\title{
Treatment of prostate cancer with intensity modulated radiation therapy (IMRT)
}

\author{
TrATAMENTO DE CÂNCER DE PRÓSTATA COM RADIOTERAPIA DE INTENSIDADE MOdULAR (IMRT) \\ Authorship: Brazilian Society of Radiology \\ Participants: Novaes PERS, Motta RT, Lundgren MSFS \\ Final Draft: March $23^{\text {th }}, 2013$
}

http://dx.doi.org/10.1590/1806-9282.61.01.008

The Guidelines Project, an initiative of the Brazilian Medical Association, aims to combine information from the medical field in order to standardize procedures to assist the reasoning and decision-making of doctors.

The information provided through this project must be assessed and criticized by the physician responsible for the conduct that will be adopted, depending on the conditions and the clinical status of each patient.

\section{Description Of the EVIDENCE COLLECTION ME- THOD}

Through the development of relevant clinical questions related to the proposed theme, we tried to present the main evidence for the safety, toxicity, effectiveness and social benefit of IMRT. The study population consisted of male patients of all ages with prostate tumors of any histological type, regardless of the stage of disease or the presence of comorbidities. For this, a systematic review of the literature was performed in primary scientific research databases (Medline - Pubmed; Embase - Elsevier; Lilacs - Bireme; Cochrane Library - Central Register of Controlled Trials). All articles available until June 15, 2013, were included. The keywords used in the search were: ("Radiotherapy, Intensity-Modulated"[Mesh]) AND "Prostatic Neoplasms"[Mesh]). The articles were selected based on critical evaluation using the instruments (scores) proposed by Jadad and Oxford; references with greater strength of evidence were used. Recommendations were prepared from discussion with the writing group, composed of three members of the Brazilian Society of Radiotherapy. The guideline was reviewed by an independent group specializing in evidence-based clinical guidelines. After completion, the guideline was made available for public consultation for 15 days and the suggestions forwarded to the writers for evaluation and consideration [possible incorporation] into the final text.

\section{DegReEs OF RECOMMENDATION AND STRENGTH OF EVIDENCE}

A. Experimental or observational studies of higher consistency.

B. Experimental or observational studies of lower consistency.
C. Case reports (non-controlled studies).

D. Opinions without critical evaluation, based on consensus, physiological studies, or animal models.

\section{Objective}

To assess whether the Intensity Modulated Radiotherapy (IMRT) should be considered as the most appropriate method of external radiation therapy for the treatment of patients with prostate cancer.

\section{Conflict of interest}

No conflict of interest informed.

\section{INTRODUCTION}

Prostate cancer $(\mathrm{PCa})$ is the sixth most common type of cancer worldwide and the most prevalent in men, accounting for about $10 \%$ of all cancer cases. In the world population, about $3 \%$ of men die from PCa (D). ${ }^{1}$ The mortality/incidence ratio of this type of cancer varies from 0.13 in North America to 0.80 in Africa, reflecting, in part, its good prognosis. The worldwide average estimated five-year survival rate is $58 \%$. Incidence rates are about six times higher in developed countries compared to developing countries. In Brazil, in 2012, 60,180 new cases of PCa were estimated and, except for non-melanoma skin tumors, this was the most common cancer among men. The figures correspond to 62 new cases per 100,000 men(D). ${ }^{2}$

$\mathrm{PCa}$ is considered a cancer of the elderly, because about three quarters of the cases throughout the world occur at 65 years of age or older. In general, the tumor has a slow growth with long doubling time, taking about 15 years to reach $1 \mathrm{~cm}(\mathbf{D}){ }^{3}{ }^{3}$ To date, research has confirmed the presence of two risk factors associated with the development of PCa: age and heredity. If a first-degree re- 
lative has the disease, the risk at least doubles. If two or more first-degree relatives have the disease, the risk increases 5-11 times $(\mathbf{D}) .^{3}$

Early detection of PCa in most cases is done through digital rectal examination or by measuring the serum concentration of prostate specific antigen (PSA). Diagnosis must be confirmed by biopsy, usually performed with a needle guided by transrectal ultrasound (D). ${ }^{4}$ Currently, serum PSA is widespread and therefore many patients have early diagnosis of PCa before presenting the classic symptoms of the disease. PCa may also be diagnosed as a result of surgical investigation or treatment of benign prostatic hyperplasia (BPH) (D). ${ }^{1}$

Once the disease is diagnosed, a prognostic analysis for prostate cancer is usually performed by combining the results of the PSA, clinical staging and histological tumor grade, in addition to the life expectancy of the patient. Clinical staging is generally based on the 2002 TNM classification by the American Joint Committee on Cancer (D). ${ }^{4}$ The TNM classification describes the extent of the primary tumor $(\mathrm{T})$, presence or absence of tumor in nearby lymph nodes $(\mathrm{N})$ and presence or absence of metastasis $(M)$. The histological grade of the tumor can be expressed using Gleason score, which is based on examination of tissue samples from the prostate, seminal vesicles and adjacent structures and, where relevant, pelvic lymph nodes. A score of 2 to 10 is given so that 2 indicates that the tumor is less aggressive and a score of 10 represents the most aggressive cancer $(\mathbf{D}){ }^{3}$

The combination of clinical classification staging, histological grade and PSA levels are widely used to assess the risk of microscopic tumor spread beyond the prostate, the risk of recurrence and the probability of success of local therapy.

From results of large studies, many nomograms using these prognostic factors were developed to assist this assessment. A nomogram is a statistical tool used to describe the likely course of disease using variables such as diagnostic findings, age, and treatment options. The definitions of degrees of disease risk (low, moderate and high) have varied slightly among the different nomograms. The most commonly used risk stratification nomogram is presented by the National Comprehensive Cancer Network (NCCN), which defines the following risk stratification:

- Low: T1-T2a, Gleason 2-6 and PSA <10ng/mL;

- Moderate: T2b-T2c, Gleason 7 or PSA 10-20ng/mL;

- High: T3a, Gleason 8-10 or PSA >20ng/mL.
Risk categories are used by the physician as an essential element for decision-making and discussion with patients about the options for local medical treatment. New independent prognostic factors are being investigated and probably the next generation of scales will incorporate other pre and post-treatment variables to predict clinical outcomes in $\mathrm{PCa}(\mathbf{D}){ }^{4}$

The most common treatments for prostate cancer include:

1. Radical prostatectomy (surgery for total removal of the prostate and surrounding structures);

2. External beam radiation therapy [including conventional radiotherapy (2D), three-dimensional conformal radiotherapy (3D-CRT), intensity modulated radiation therapy (IMRT), and three-dimensional radiotherapy or intensity modulated radiation therapy directed to treatment target - Image Guided Radiotherapy (IGRT)];

3. Brachytherapy;

4. Cryotherapy;

5. Androgen suppression therapy (hormone therapy).

The goals of treatment are to prevent death and disability due to PCa, minimizing complications related to the interventions.

\section{TECHNOLOGY DESCRIPTION}

Radiotherapy is the specific use of high-energy ionizing radiation that can use $\mathrm{X}$-ray, gamma ray, or proton treatment beams, as well as other sources to treat cancer. Radiation may come from an external source (external radiotherapy) or from a source located close to the tumor, placed inside the body (brachytherapy).

In recent years, the planning and the execution of radiotherapy have gone through a significant process of change. This change results mainly from continued advances in the incorporation of imaging methods and new "hardware and software" to planning, which have enabled the continuous improvement of the methods for release of radiation, dosage accuracy and implementation of radiotherapy, combined with a continuous investment in education and training for professionals who perform the investigations $(\mathbf{D}) .^{5}$

One method widely used is the three-dimensional conformal radiotherapy (3D-CRT). This is an advanced form of external radiation therapy that uses the computer to create a three-dimensional image of the tumor, allowing multiple radiation beams of uniform intensity to be conformed exactly to the contour of the target treatment area (area to be treated plus a safe margin) $(\mathbf{B}){ }^{6}$ 
Intensity Modulated Radiation Therapy (IMRT) is an advanced technique of radiation dose release. Developed in the late 1990s, IMRT not only allows radiation to be shaped to the contour of the target area, but also uses multiple angular radiation beams and non-uniform intensities. IMRT is an evolution of conformal radiotherapy, since it is able to modulate the treatment beam, providing greater dose intensity at the area of interest and sparing areas where intensity is not desired. With this, it is possible to irradiate irregular tumors; tumors with concave features; and tumors with complex morphology that "surround" normal structures, without causing excessive exposure of surrounding tissues to radiation. The objective is to conform the radiation to the shape of the target volume as much as possible, in an attempt to further protect adjacent structures $(\mathbf{D}) .{ }^{4,7}$ IMRT techniques are significantly more complex than other traditional forms of radiation, including conformal.

The radiation dose is designed to conform to the three-dimensional shape of the tumor by modulating or controlling the intensity of sub-components in each radiation beam. Therefore, high radiation dose is used in the target tumor while the expectation is to reduce the exposure of normal surrounding tissues to radiation, seeking to reduce treatment toxicity.

IMRT planning is more complex and challenging than the planning of conformal radiotherapy. In both cases, the specialist in radiotherapy indicates specific targets (target tumor, nodular elective areas) and avoided structures (rectal wall, bladder, coxofemoral region, intestines, etc). In conformal radiation, the arrangement of the beams is shaped so that there are generous field margins to compensate for daily configuration variations and physical characteristics of the beam itself. IMRT planning, on the other hand, requires dose specifications to be defined both for the target organ and the structures that must be protected, establishing tolerance restrictions according to the importance of protection needed for that specific organ under evaluation. The planning software creates a set of standards for modulation and angulation of each beam seeking to reach the doses prescribed by the doctor. This process is known as inverse planning $(\mathbf{D}){ }^{7}$

In clinical practice, IMRT application requires several steps, from planning to procedure completion $(\mathbf{B}):^{6}$

1. Image acquisition: the images, usually $\mathrm{CT}$ scan of the area to be radiated, must be obtained in the same position that the patient will undergo treatment, with all the parts used for immobilization. Serial sections of the area to be irradiated are taken, using thin thickness predetermined by a doctor. The criteria for rectal and bladder filling are given to the patients before taking the images, and the machine used for acquiring them should be devoted to radiation therapy. If the test is carried out using a non-dedicated machine, a rectifier board for decubitus support must be manufactured to ensure reproducibility of positioning during image acquisition, similar to that of the time of treatment.

2. Image transfer: after image acquisition, they are transferred to the computer that will perform the treatment's calculations.

3. Structure outlining: careful outlining of the irradiation volumes and organs at risk is necessary, with the aid of computed tomography and other image methods (usually MRI), so that the prescribed dose of radiation can reach the target organ, affecting the normal surrounding tissues as little as possible.

4. Assignment of restrictions and permissions: organs and structures that will be positively valued or with less intensity are defined, as well as dose prescription and constraints. Next, the technician prepares the planning according to these settings.

5. Physical planning: a personalized treatment plan is prepared so that the requirements of 'target', 'dose' and 'dose constraint of the surrounding structures' are respected. 3D CRT scan images of the patient in conjunction with computerized calculation of doses are used for this purpose.

6. Analysis of the dose per volume and acceptance of the calculated plan: the plan calculated by the technician is evaluated, with special attention to the analysis of the dose-volume histogram, accepting it or rejecting it, according to the specifications of each organ in each specific case.

7. Data transfer to the therapy equipment: the data is transferred to the machine where the patient will undergo treatment, and the radiation technician evaluates all predetermined parameters.

8. Verification of patient positioning and prescriptions: the patient is positioned and positioning checks are made. After all the technical parameters on the screens are confirmed, treatment is initiated. The whole course of treatment is monitored and supervised by cameras aiming at ensuring the immobilization of the patient during movement of the machine. Treatment involves reproduction of the therapeutic plan, day to day and field to field, with the correct positioning of the patient and the precise location of the target organ. 


\section{IS THERE A DIFFERENCE IN THE EFFICACY OF TREATMENT OF PROSTATE TUMORS WITH IMRT?}

There are few data that examine the comparative effects of treatments for PCa. Data suggest that these interventions have comparable cure rates; however, they differ in terms of characteristics of side effects. As there is no single approach considered "reference standard", patients and physicians have difficulty in choosing between treatment options to determine the best therapeutic approach $(\mathbf{D})^{4}(\mathbf{B}){ }^{8}$

There is evidence that surgery, radiotherapy and brachytherapy are effective approaches for disease control (B). ${ }^{9}$

The importance of maintenance doses equal to or greater than 80Gy, particularly in patients with high Gleason score, is pointed out to obtain better results in terms of reduction of biochemical failure risk, progression to metastatic disease and overall death $(\mathbf{B}) .^{10}$

One study showed a linear improvement in biochemical control with dose escalation to 80Gy, noting that better controls can be expected beyond that dose (B). ${ }^{11}$

Authors found evidence indicating that surgically treated patients tend to have more genitourinary symptoms and sexual dysfunction, while irradiated patients tend to suffer more gastrointestinal symptoms (B). ${ }^{8}$ Another study that only evaluated the ability to maintain erectile function and included 54 trials (5.000 patients) revealed that there is a greater chance of preserving erectile funtion using radiation therapy compared to all surgical interventions (radical prostatectomy, nerve sparing surgery or cryotherapy) $(\mathbf{B}) .{ }^{11}$

\section{Recommendation}

There is no data showing efficacy of the different prostate cancer treatments, only data that suggest comparable cure rates; nevertheless, therapies differ in terms of characteristics of side effects.

\section{IS THERE LESS TOXICITY WHEN IRRADIATING PROS- TATE TUMORS WITH IMRT COMPARED TO CON- FORMAL OR CONVENTIONAL RADIOTHERAPY?}

The main adverse effects of radiation therapy result from radiation effects on surrounding tissues (gastrointestinal, genitourinary and sexual organs). The toxic effects are classified as acute (when they occur within 90 days after initiation of treatment) or late. Gastrointestinal initial symptoms include abdominal cramps, tenesmus (painful sensation in the lower abdomen or in the anal area with a constant feeling of the need to pass stool, but without almost any relief), urgency and increased stool frequency that are usually controlled with antidiarrheal medication. Delayed gastrointestinal effects include urgency, change in stool frequency, and mucus discharge or rectal mucosal blee- ding, which may require specialized treatment. Moderate to severe genitourinary effects are caused by the irritability of the detrusor muscle or urothelial inflammation, resulting in urgency, frequency changes and dysuria (difficulty urinating). Delayed genitourinary effects include obstruction of the bladder neck or urethra, causing urgency and sometimes acute urinary retention. In addition to these, erectile dysfunction is an effect often observed and its mechanism is not yet fully understood (D). ${ }^{4}$

In Brazil, as described in the list of health procedures and events of the Brazilian Supplemental Health System (Agência Nacional de Saúde Suplementar, ANS), brachytherapy, conventional radiotherapy (2D) and conformal radiotherapy (3D-CRT) are mandatory coverage techniques for PCa by private health plans, in accordance with the characteristics of each plan (D). ${ }^{12,13}$

Based on clinical experience with radiotherapy complications, a standardized dose limit per volume of normal organ has been created, namely Quantitative Analysis of Normal Tissue Effects in the Clinic (Quantec) (B), ${ }^{14}$ recommended throughout the world today as a practical guide for conducting radiotherapy in all parts of the body. One can only know this quantification of dose based on three-dimensional planning.

This renders the $2 \mathrm{D}$ conventional radiotherapy obsolete due to inaccuracy in locating the target, requiring large margins to avoid error, and also the impossibility of determining the dose received by normal organs. Therefore, modern radiotherapy for curative treatment of prostate cancer using external radiation therapy requires the use of three-dimensional techniques such as IMRT and conformal.

Experience with this type of treatment is increasing in our midst, but recent studies have indicated that this form of dose administration can be improved in patients with prostate cancer, especially in high-risk lesions and in advanced disease.

Several authors have shown that dose escalation, a technique in which the amount of ionizing radiation delivered to the tumor is equal to or greater than $78 \mathrm{~Gy}$, is related to increased local control of disease, but conformal technique does not allow such high doses, with dosimetric safety constraints to organs at risk, especially rectum, bladder and head of femur $(\mathbf{B})^{10,15}(\mathbf{D}) .^{16,17}$

\section{Recommendation}

Conformal radiation therapy is increasingly being replaced, since this technique does not allow higher doses to be achieved, with dose safety constraints per volume of normal organ being maintained. IMRT with radiation dose greater than or equal to $78 \mathrm{~Gy}$ is recommended. 


\section{IS IRRADIATION OF PATIENTS WITH PROSTATE CAN- CER USING IMRT A SUPERIOR TECHNIQUE COM- PARED TO CONFORMAL RADIATION THERAPY?}

The search for evidence to enable the answer to this question was performed in six scenarios:

1. Localized disease;

2. Locally advanced disease;

3. Pelvic lymph node radiotherapy;

4. Radiotherapy after prostatectomy;

5. Hypofractionated Regimens;

6. Dose escalation.

\section{LOCALIZED DISEASE}

Radiotherapy is used as definitive treatment, in high doses, in patients with localized prostate cancer, resulting in local control of the disease $(\mathbf{D})^{17}(\mathbf{B}) .^{18}$

The recommended dose for curative treatment of localized prostate cancer is well established as $72 \mathrm{~Gy}$. Patients with high-risk tumors should receive higher dose, usually around 78Gy $(\mathbf{D}) \cdot{ }^{19,20}$

A study comparing radical prostatectomy with high-dose radiotherapy using IMRT (doses equal to or greater than 72Gy) showed that for patients with localized high-risk prostate cancer there was an increase in biochemical relapse-free survival. The difference was $38.4 \%$ versus $62.2 \%$, favoring $\operatorname{IMRT}(\mathbf{B}){ }^{21}$

Authors analyzed eleven studies involving 4,559 patients treated with IMRT and conformal radiotherapy $(\mathbf{D}){ }^{22}$ One study showed favorable results in favor of IMRT in respect of biochemical failure-free survival at five years (IMRT $=74.1 \%$ versus conformal $=60.4 \% ; \mathrm{p}<0,0001)(\mathbf{B}) ;{ }^{23}$ and another study showed no significant difference in clinical recurrence-free survival at five years (IMRT $=90 \%$ versus conformal $=72 \% ; p=0.07)(B) .{ }^{24}$ None of the studies, however, showed differences in survival for specific disease. With regard to acute and delayed adverse effects, both of gastrointestinal and genitourinary nature, although there were slight differences among the studies, the results of toxicity in patients treated with IMRT were better compared to conformal radiation $(\mathbf{D}) .^{22}$

\section{Recommendation}

IMRT has dosimetric advantages over conformal radiotherapy in patients with localized disease.

\section{LOCALLY ADVANCED DISEASE}

The combination of radiotherapy and androgen deprivation is considered standard therapy for locally advanced prostate cancer. This scenario classically includes large tumors, stage $\mathrm{T} 3$ and some $\mathrm{T} 4$, which require approximate doses of 78Gy or more for control.

Patients with localized prostate cancer who received radiation doses as high as 81Gy using IMRT achieved excellent local control in 10 years and the findings indicate that IMRT in high doses was well tolerated $(\mathbf{B}){ }^{25}$

A study comparing conformal radiotherapy and IMRT showed that radiation dose escalation, which is possible using IMRT (doses greater than 80Gy), was associated with less risk of biochemical failure, metastatic disease and overall mortality, with no increase in genitourinary and gastrointestinal toxicity. The use of IMRT enabled increase in radiation dosage (dose escalation), with reduced toxicity to normal surrounding tissue, increasing the local biochemical control of the disease, as well as distant metastatic disease-free survival, and reducing the specific cancer mortality rate $(\mathbf{B}) .^{10}$

The evaluation of new treatments such as IMRT, radiation therapy with protons and conformal radiotherapy, conducted in patients registered in the SEER (Surveillance Epidemiology and End Results) database, showed that IMRT was associated with lower gastrointestinal morbidity and fewer femoral fractures compared to conformal radiotherapy $(\mathbf{B}){ }^{26}$

In a study comparing the benefits of IMRT against conformal radiation therapy, both used in combination with brachytherapy, the results showed reduced rectal toxicity grade $2-3$ (rectal bleeding) with IMRT (7 versus $11 \%$ ), less acute urinary toxicity and better quality of life. Thus, there is a significant reduction in gastrointestinal toxicity with IMRT $(\mathbf{B}){ }^{27}$

\section{Recommendation}

Patients with localized prostate cancer who received radiation doses higher than 80Gy using IMRT achieved excellent control, lower risk of biochemical failure, metastatic disease and overall mortality without increasing gastrointestinal and genitourinary toxicity.

\section{Pelvic lymph node irRadiation}

Irradiation of pelvic lymph nodes arouses many controversies in the management of prostate tumors.

Dosimetric studies showed superiority of IMRT compared to conformal radiotherapy in some points, such as dose escalation, lower irradiation to the rectum and intestines, and better coverage of pelvic lymph nodes by the prescription isodose $(\mathbf{B}) .^{28-31}$

These studies from several institutions used computerized planning systems that allowed the recognition of target volumes and organs at risk. They produced dose-volume histograms that allow for a proper analysis of the dose distribution in normal tissues and choice of the best technique. 
Other authors compared 68 patients undergoing single radiation therapy, delivered to the prostate up to $76 \mathrm{~Gy}$ using conformal radiotherapy, and 45 patients undergoing IMRT with doses of 54 and 76Gy, giving respective coverage of the pelvic lymph nodes and prostate. They found a cumulative incidence of grade 2 rectal toxicity in two years, $6 \%$ for patients treated with IMRT and $21.2 \%$ for those treated with 3D-CRT $(\mathrm{p}=0.06)(\mathbf{B}){ }^{31}$

Other studies comparing results of toxicity due to pelvic irradiation in patients undergoing conformal radiotherapy versus IMRT revealed no significant difference in urinary ( 12.3 versus $6.6 \% ; \mathrm{p}=0.19)$ and rectal ( 8.6 versus $3.2 \%$; $\mathrm{p}=0.14$ ) toxicity degree $\geq 2$; for intestinal toxicity, the decrease was quite significant ( 22.2 versus $6.6 \% ; \mathrm{p}=0.004$ ), and treatment discontinuation occurred more among patients treated with conformal radiotherapy (11 out of 81) compared to those treated with IMRT ( 2 out of 91$), p=0.006$. Univariate analysis showed correlation with significant values for intestinal volume receiving $45 \mathrm{~Gy}$ doses, as well as for previous pelvic-abdominal surgery $(\mathbf{B}){ }^{33,34}$

Irradiation of pelvic lymph nodes, regardless of the technique used for teletherapy, showed a survival advantage in most studies arising from the addition of hormone deprivation therapy. Nevertheless, it should be kept in mind that the risk of lymph node involvement also increases the risk of distant metastases, which can reduce or cancel any potential benefit obtained from the irradiation of pelvic lymph nodes. Therefore, this may be indicated according to the preference of the treating physician in high-risk patients $(\mathbf{D}) .{ }^{19}$

Most studies compared conformal radiation therapy and IMRT as treatments for prostate only. As explained above, the role of pelvic lymph node irradiation in prostate cancer remains controversial and, if used, pelvic irradiation may exacerbate gastrointestinal and genitourinary adverse effects, both acute and delayed, due to an increase in volume of irradiated normal tissue versus prostate irradiation only $(\mathbf{D}) \cdot{ }^{17}$ The comparison of results of patients considered high-risk undergoing conformal radiotherapy with doses between 46 and 50Gy in pelvic lymph nodes, plus 20Gy booster therapy, and patients treated with IMRT at doses of 56Gy on pelvic lymph nodes plus 24Gy booster showed that the group treated with higher doses, enabled by IMRT, presented lower risk for biochemical failure, metastases and overall mortality after univariate analysis $(\mathbf{D}){ }^{7}$

\section{Recommendation}

The irradiation of pelvic lymph nodes using computerized planning systems, with adequate analysis of the dose distribution in normal tissues, indicates that IMRT is the best technique.

\section{RADIATION AFTER PROSTATECTOMY}

The indication of postoperative external radiotherapy may be considered as adjuvant therapy in patients with risk factors for recurrence or as a rescue option after any biochemical failure. In this respect, it is recognized as the only curative option and has shown significant growth in clinical practice, to the point of representing, in some centers, percentages as high as those of patients undergoing primary irradiation.

Authors compared patients undergoing conformal radiotherapy with IMRT as adjuvant or rescue therapy delivered to lymph nodes and prostate bed, finding less acute urinary and lower digestive toxicity, despite no statistically significant values. However, they observed a significant reduction of upper gastrointestinal toxicity in favor of patients undergoing IMRT as well as less treatment interruptions $(2.2$ versus $13.6 \%)(\mathbf{B}) .^{33}$

Higher gastrointestinal toxicity was observed in patients who were treated with previous prostatectomy compared to patients undergoing primary prostate irradiation (B). ${ }^{34}$ From this finding, we can conclude that radiotherapy techniques that provide less toxicity are the most indicated.

There are data showing no significant difference in gastrointestinal acute toxicity grade $\geq 2$ when patients were treated with IMRT compared to conformal radiotherapy ( $\mathrm{p}=0.14)$. In terms of delayed toxicity ( 5 years), occurrences were observed in $10.2 \%$ of patients undergoing conformal radiation therapy and in $1.9 \%$ of patients treated with IMRT $(\mathrm{p}=0.02)(\mathbf{B}){ }^{35}$

Rescue radiation therapy is offered to patients with high levels of PSA after radical prostatectomy. It is consensus that PSA levels considered as failure following radical surgery is in the range of $0.2-0.5 \mathrm{ng} / \mathrm{mL}$.

A study reported results after IMRT used as rescue therapy in cases of biochemical failure, compared with non-modulated techniques in terms of toxicity. The main conclusion was that IMRT was associated with a statistically significant and substantial decrease in delayed gastrointestinal toxicity, even at doses higher than 70Gy compared to doses of less than 70Gy using conformal radiotherapy $(\mathbf{B}) .^{35}$

Although there is no evidence from randomized trials that higher doses provide better controls as rescue or adjuvant therapy, there is indirect evidence supporting dose escalation in patients treated with prostatectomy, and the general concern of using doses >70Gy was partially relieved $(\mathbf{B}) .^{35}$

A publication presented results of the effectiveness of IMRT and conformal radiotherapy in the treatment of 
PCa after prostatectomy, analyzing data from the SEER database. The analysis involved 1,014 patients, of whom 457 received IMRT and 557 received conformal radiotherapy. Both groups had similar results for morbidity and disease control, demonstrating that the benefit of IMRT in this situation is uncertain (B). ${ }^{36}$

\section{Recommendation}

The use of IMRT as rescue therapy in cases of biochemical failure, compared to non-modulated techniques, showed advantages in terms of toxicity. The main conclusion was that IMRT was associated with a statistically significant and substantial decrease in delayed gastrointestinal toxicity, even when doses higher than 70Gy were delivered compared to doses of less than 70Gy using conformal radiotherapy.

\section{Hypofractionated regimens}

The best regimen for external radiotherapy in prostate cancer is still subject to questioning. Several recent publications have suggested that the alpha/beta ratio for prostate is low, in the range 1-3Gy. If this is true, dosage regimens using fewer fractions with higher dose/fraction intensity could improve the results. Hypofractioning with higher daily fractions of $2.5 \mathrm{~Gy}$ can maintain higher bioequivalent tumor doses, without causing increased acute and delayed side effects, reducing the number of treatment visits for the end of radiotherapy, increasing capacity and reducing treatment costs. In institutions where the operational capacity is limited, hypofractionated radiotherapy regimens have enormous social impact.

Authors published the results of studies comparing hypofractionated external radiotherapy versus conventional radiotherapy in patients with localized prostate cancer, and concluded that hypofractionated radiotherapy is not superior to conventional radiotherapy in these patients, also showing acute gastrointestinal toxicity slightly higher (D). ${ }^{38}$ Other authors studied the toxicity of hypofractionated radiotherapy versus conventional fractionation, including 217 patients treated with conformal radiotherapy comparing $69 \mathrm{~Gy}$ ( 23 fractions of $3 \mathrm{~Gy}$ ) versus 78Gy (39 fractions of $2 \mathrm{~Gy}$ ). The hypofractioning scheme using conformal radiotherapy with high biologically effective dose is feasible in prostate cancer and is well tolerated with minimal severe acute toxicity $(\mathbf{B}){ }^{39}$

The dosage regimen, with consecutive fractions at doses of $3 \mathrm{~Gy} /$ day was also compared to other regimens reported in the literature using daily fractions between 2.7 and $6.5 \mathrm{~Gy}$, which showed similar results $(\mathbf{B}) .^{39}$

\section{Recommendation}

Hypofractioning, with higher daily fractions of $2.5 \mathrm{~Gy}$, can maintain higher bioequivalent tumor doses without causing increased acute and delayed side effects. Hypofractionated radiation therapy was not superior to conventional radiation therapy in these patients, and led to slightly higher acute gastrointestinal toxicity.

\section{Dose escalation}

What dose of external radiation is high enough for prostate cancer? In a study carried out to compare four different doses delivered to prostate isocenter $(<70 \mathrm{~Gy}, 70$ 74,9Gy, 75-79,9Gy and >80Gy), which involved 1,530 patients, the authors concluded that doses higher than or equal to $80 \mathrm{~Gy}$ are recommended for most men with prostate cancer $(\mathbf{B}) .{ }^{40}$ The study showed a direct relationship of dose-response, which had already been suggested in previous reports and was confirmed by other authors in subsequent publications (B)..$^{40-43}$

The possibility to optimize dose distribution with IMRT led to the development of studies delivering doses higher than 78-80Gy. Authors have published the results of 1,002 patients treated at doses of 86.4Gy using IMRT technique of 5 to 7 fields. With median follow-up of 5.5 years, the survival rate in seven years free of biochemical relapse was 98.8, 85.6 and $67.9 \%$ for low, moderate and high-risk patients, respectively $(\mathrm{p}<0,001)$, while metastasis-free survival reached $99.4,94.1$ and $82 \%$, respectively $(\mathrm{p}<0,001)$. In the multivariate analysis, tumor stage $(T)$, Gleason score and the percentage of positive biopsies were the predictors of distant metastases and cancer-specific mortality. The rates of side effects grade 3 , both gastrointestinal and genitourinary, were 0.7 and $2.2 \%$, respectively. The findings indicate that dose escalation with IMRT is feasible, with excellent clinical results and acceptable toxicity $(\mathbf{D}) .{ }^{17}$

\section{Recommendation}

Doses greater than or equal to $80 \mathrm{~Gy}$ are recommended for most men with prostate cancer, and there is a direct relationship between dose and response. The findings indicate that dose escalation (86.4Gy) with IMRT is feasible, with excellent clinical results and acceptable toxicity.

\section{EVIDENCE SUMMARY}

IMRT is a viable therapeutic option, endorsed by the studies mentioned in this guideline, and reinforced by the principle of precaution, so that it is ethical to recommend a treatment with fewer expected adverse effects over another with more effects. Although advantages in overall 
survival and biochemical failure-free survival have not been demonstrated, reductions in genitourinary and gastrointestinal toxicity, both acute and late, and the consequent improvement in quality of survival justify its use.

The possibility of dose escalation is clearly feasible with IMRT and this is a trend to improve results in locally advanced tumors. The development of hypofractionated radiotherapy programs, shortening the total time of therapy administration, is of great social value, reducing waiting times for the start of radiation therapy, especially in high-demand healthcare services.

\section{References}

1. National Institute for Clinical Excellence (NICE). Prostate Cancer: Diagnosis and Treatment. Full Guideline CG 58. 2008. Disponível em: http://guidance. nice.org.uk/CG58/Guidance/pdf/English.

2. Brasil. Ministério da Saúde. Secretaria de Atenção à Saúde. Instituto Nacional de Câncer. Coordenação de Prevenção e Vigilância de Câncer. Estimativas 2008.

3. Heidenreich A, Aus G, Bolla M, Joniau S, Matveev V, Schmid H, Zattoni F. Guía de la EAU para el Cáncer de Próstata. Actas Urológicas Españolas 2009; 33(2):113-26.

4. Pearson S, Ladapo J, Prosser L. Intensity Modulated Radiation Therapy (IMRT) for Localized Prostate Cancer. Institute for Clinical and Economic Review. Final Appraisal Document 2007.

5. Boyer A, Butler B, Dipetrilo T, Engler M, Fraass B, Grant W, et al. IntensityModulated Radiotherapy: Current Status and Issues of Interest. Int. J. Radiati Oncol Biol Phy. 2001;51(4):880-914

6. Van Den Steen D, Hulstaert F, Camberlin C. Intensity-modulated radiotherapy (IMRT). Health Technology Assessment (HTA). Brussels: Belgian Health Care Knowledge Centre (KCE); 2007. KCE reports 62C (D/2006/10.273/34).

7. Hong $T$, Ritter M, Harari P. Intensity-modulated radiation therapy: emerging cancer treatment technology. British Journal of Cancer 2005;92:1819-24.

8. Wilt T, McDonald R, Rutks I, Shamliyan T, Taylor B, Kane R. Systematic Review: Comparative Effectiveness And Harms Of Treatments For Clinically Localized Prostate Cancer. Annals Of Internal Medicine 2008;148(6):435-48.

9. Nilsson S, Norlen BJ, Widmark A. A systematic overview of radiation therapy effects in prostate cancer. Acta Oncol. 2004;43(4):316-81.

10. Pahlajani N, Ruth KJ, Buyyounouski MK, Chen DY, Horwitz EM, Hanks GE, Price RA, Pollack A. Radiotherapy doses of 80 Gy and higher are associated with lower mortality in men with Gleason score 8 to 10 prostate cancer. Int J Radiat Oncol Biol Phys 2012;82(5):1949-56

11. Robinson JW, Moritz S, Fung T. Meta-analysis of rates of erectile function after Treatment of localized prostate carcinoma. Int J Radiat Oncol Biol Phys. 2002;54(4):1063-8.

12. Agencia Nacional de Saúde Suplementar. Radioterapia de Intensidade Modulada (IMRT) para o Câncer de Próstata. Informe ATS 2009.

13. Brasil. Ministério da Saúde. Agência Nacional De Saúde Suplementar (ANS). Resolução Normativa - $\mathrm{RN}$ n$^{\circ} 262$, de 02 de agosto de 2011, que atualiza o Rol de Procedimentos e Eventos em Saúde.

14. Marks LB, Haken RKT, Martel MK. Quantitative analyses of normal tissue effects in the clinic. Int J Radiat Oncol Biol Phys 2010; 76 (3 Supp):1-160.

15. Michalski JM, Yan Y, Watkins BD, Walter B, Winter K, Galvin JM, et al. Preliminary analysis of 3D-CRT $v$ s. IMRT on the high dose arm of RTOG 0126 prostate cancer trial: toxicity report. Abstracts of the $53^{\text {rd }}$ Annual ASTRO meeting.

16. Wolff JM, Mason M. Drivers for change in the management of prostate cancer - guidelines and new treatments techniques. BJU Int. 2012; Suppl 06:33 AM-41.

17. Hayden AJ, Catton C, Pickles T. Radiation therapy in prostate cancer: a riskadapted strategy. Curr Oncol 2010; suppl 2:S18-24

18. Spratt DE, Pei X, Yamada J, Kollmeier MA, Cox B, Zelefsky MJ. Long-term survival and toxicity in patients treated with high-dose intensity modulated radiation therapy for localized prostate cancer. Int J Radiat Oncol Biol Phy. 2013;85(3):686-92.

19. Hayden AJ, Martin JM, Kneebone AB, Lehman M, Wiltshire KL, Skala M, et al. Australian \& New Zealand Faculty of Radiation Oncology Genito-Urinary
Group: 2010 consensus guidelines for definitiveexternal beam radiotherapy for prostate carcinoma. Journal of Medical Imaging and Radiation Oncology 2010;54:513-25.

20. Brain JM, Paul D, Chow JH, et al. ACR Appropriateness Criteria Definitive External Beam Irradiation in Stage T1 and T2 Prostate Cancer. American Journal of clinical Oncology 2011; 34(6): 636-647

21. Aizer AA, Yu JB, Colberg JW, et al. Radical prostatectomy vs. intensitymodulated radiation therapy in the management of localized prostate adenocarcinoma. Radiother Oncol 2009; 93(2): 185-91

22. Bauman G, Rumble RB, Chen J, Loblaw A, Warde P and members of the IMRT indications expert panel. Guidelines. Intensity-modulated radiotherapy in the management of prostate cancer. Clinical Oncology 2012; 24: 461-73.

23. Vora SA, Wong WW, Schild SE et al. Analysis of biochemical control and prognostic factors in patients treated with either low-dose three dimensional conformal radiation therapy or high-dose intensity-modulated radiotherapy for localized prostatic cancer. Int J Radiat Oncol Biol Phys 2007; 68(4): 1053-8

24. Al-Mangani A, heemsbergen WD, Peeters STH et al. Role of intensity modulated radiotherapy in reducing toxicity in dose escalation for localized prostate cancer. Int J Radiat Oncol Biol Phys 2009; 73(3): 685-91

25. Alicikus ZA, Yamada Y, Zhang Z, et al. Ten-year outcomes of high-dose, intensity-modulated radiotherapy for localized prostate cancer. Cancer 2011; 117(7):1429-1437.

26. Sheets NC, Goldin GH, Meyer AM, et al. Intensity-modulated radiation therapy, proton therapy, or conformal radiation therapy and morbidity and disease control in localized prostate cancer. JAMA 2012; 307(15): 1611-1620.

27. Forsythe K, Blacksburg S, Stone N, Stock RG. Intensity-modulated radiotherapy causes fewer side effects than three-dimensional conformal radiotherapy when used in combination with brachytherapy for the treatment of prostate cancer. Int J Radiat Oncol Biol Phys 2012; 83(2):630-635.

28. Ashman JB, Zelefsky MJ, Hunt MS, Leibel SA, Fuks Z. Whole pelvic radiotherapy for prostate cancer using $3 \mathrm{D}$ conformal and intensity-modulated radiotherapy. Int J Radiat Oncol Biol Phys 2005; 63(3):765-71.

29. Wang-Chesebro A, Xia P, Coleman J, Akazawa C, Roach M. Intensitymodulated radiotherapy improves lymph node coverage and dose to critical structures compared with three-dimensional conformal radiation therapy in clinically localized prostate cancer. Int J Radiat Oncol Biol Phys 2006; 66(3):654-62.

30. Guckenberger M, Baier K, Richter A, Vordermark D, Flentje M. Does intensity modulated radiation therapy (IMRT) prevent additional toxicity of treating the pelvic lymph nodes compared to treatment of the prostate only? Radiat Oncol 2008;03:03 AM.

31. Bayouth JE, Pena J, Culp L, Brack C, Sanguineti G. Feasibility of IMRT to cover pelvic nodes while escalating the dose to the prostate gland: dosimetric data on 35 consecutive patients. Med Dosim 2008 Autumn; 33(3):180-90.

32. Sanguineti G, Cavey ML, Endres EJ, Franzone P, Barra S, Parker BC, et al. Does treatment of the pelvic nodes with IMRT increase late rectal toxicity over conformal prostate-only radiotherapy to 76 Gy? Strahlenther Onkol 2006;182(9):543-9.

33. Alongi F, Fiorino C, Cozzarini C, Broggi S, Perna L, Cattaneo GM, et al. IMRT significantly reduces acute toxicity of whole-pelvis irradiation in patients treated with post-operative adjuvante or salvage radiotherapy after radical. Radiother Oncol 2009;93(2):207-12.

34. Fiorino C, Alongi F, Perna L, Broggi S, Cattaneo GM, Cozzarini C, et al. Dose-volume relantionships for acute bowel toxicity in patients treated with pelvic nodal irradiation for prostate cancer. Int J Radiat Oncol Biol Phys 2009;75(1):29-35

35. Goenka A, Magsanoc JM, Pei X, Schechter M, Kollmeier M, Cox B, et al. Improved toxicity profile following high-dose post prostatectomy salvage radiation therapy with intensity-modulated radiation therapy. Eur Urol 2011;60(6):1142-8

36. Goldin GH, Sheets NC, Meyer AM, et al Comparative effectiveness of Intensity-modulated radiotherapy and conventional conformal radiotherapy in the treatment of prostate cancer after radical prostatectomy. JAMA Intern Med 2013;173(12):1136-43

37. Aneja S, Pratiwadi RR, Yu JB. Hypofractionated radiation therapy fot prostate cancer: risks and potential benefits in a fiscally conservative health care system. Oncology 2012; 26(6):1-9.

38. Botrel TE, Clark O, Pompeo ACL, et al. Hypofractionated external-beam radiation therapy (HEBRT) versus conventional external-beam radiation (CEBRT) in patients with localized prostate cancer: a systematic review and meta-analysis. Core Evidence Dovepress 8: 2013. 
39. Viani GA, Silva LBG, Silva BB, et al. Acute toxicity in prostate cancer with conventional and hypofractionated treatment. Radiation Oncology 2013; 8:94-100.

40. Eade TN, Hanlon AL, Horwitx EM, et al. What dose of external-beam radiation is high enough for prostate cancer ? Int J Radiat Oncol Biol Phys 2007; 68(3) 682-9.

41. Beckendorf V, Guerif S, le Prise E, et al. The GETUG 70Gy vs. 80Gy randomized trial for localized prostate cancer: feasibility and acute toxicity. Int J Radiat Oncol Biol Phys 2004; 60:1065-65
42. Peeters ST, Heemsbergen WD, Koper PC, et al. Dose-response in radiotherapy for localized prostate cancer: results of the Dutch multicenter randomized phase III trial comparing 68Gy of radiotherapy with 78Gy.J Clin Oncol 2005; 23: 1990-96.

43. Dearnaley DP, Sydes MR, Graham JD, et al. Escalated-dose versus standarddose conformal radiotherapy in prostate cancer: First results from the MRC RT01 randomized controled trial. Lancet Oncol 2007; 8:475-87.

44. Kuban DA, Ptretzer SL, Dong L et al; long-term results of the M D Anderson randomized dose-escalation for prostate cancer. Int $\mathrm{J}$ radiat Oncol Biol Phys $2008 ; 70: 67-74$ 